STEADY-STATE AND TIME-DEPENDENT LUMINESCENCE OF $\mathrm{UO}_{2} \mathrm{MoO}_{4}$

\title{
W.M.A. SMIT
}

Physical Laboratory, University of Utrecht, P.0. Box 80.000, 3508 TA Utrecht, The Netherlands

Luminescence spectra of $100, \mathrm{MoO}$ between 4.2 - $50 \mathrm{~K}$ were studied. The $0-0$ line splitting is explained. Decay functions were derived reproducing the temperature dependence of the trap emissions. The rate of energy transfer among intrinsic centres and to trap centres is shown to be of similar magnitude.

\section{INTRODUCTION}

In $\mathrm{UO}_{2} \mathrm{MoO}_{4}$ (space-group $\mathrm{P}_{2} / \mathrm{C}^{-} c_{2 h}^{5}, z=4$ ) the uranyl groups have $C_{1}$ site symmetry [1]. The lowest excited state of the symmetric uranyl group is ${ }_{\mathrm{g}}[2]$, but due to correlation field splitting $(Z=4)$ we expect in $\mathrm{UO}_{2} \mathrm{MoO}_{4}$ eight levels: $2 \mathrm{~A}_{\mathrm{g}}+2 \mathrm{~B}_{\mathrm{g}}+2 \mathrm{~A}_{u}+2 \mathrm{~B} u$. This was investigated by studying the luminescence spectra. The temperature dependence of the trap decay times is studied with regard to energy migration. The experimental set-up is described in Ref. 3.

\section{RESULTS AND DISCUSSION}

The steady-state emission mainly results from traps which appear to be distorted uranyl groups (see Table 1). The intrinsic emission, observed at 50 ns after the pulse, shows progressions in the $A_{\mathrm{g}}$ correlation field components of the $\mathrm{UO}_{2}$ stretching modes (see Table 1) |3].

Table 1. Observed emission lines for the intrinsic centres and the two more intensive trapcentres.

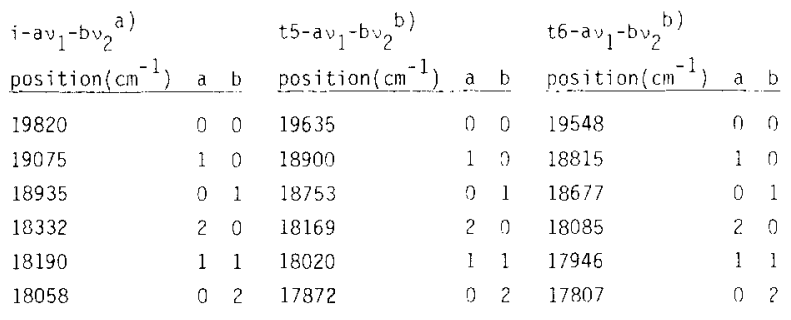

a) $i=$ intrinsic centre, $v_{1}, v_{2}=A_{g}$ correlation field component of $\mathrm{UO}_{2}$ symmetrical and asymmetrical stretching mode, respectively.

b) $\mathrm{t} 5, \mathrm{t} 6=$ trap centres 5 and 6 (see Ref. 3). 
The lineshape of the intrinsic 0 - 0 line could be interpreted in terms of four component lines, representing the transitions to the $2 \mathrm{~A}_{\mathrm{u}}+2 \mathrm{~B} u$ excited levels, in accordance with the observed temperature dependence of the emission lineshape (see fig. 1)[3].

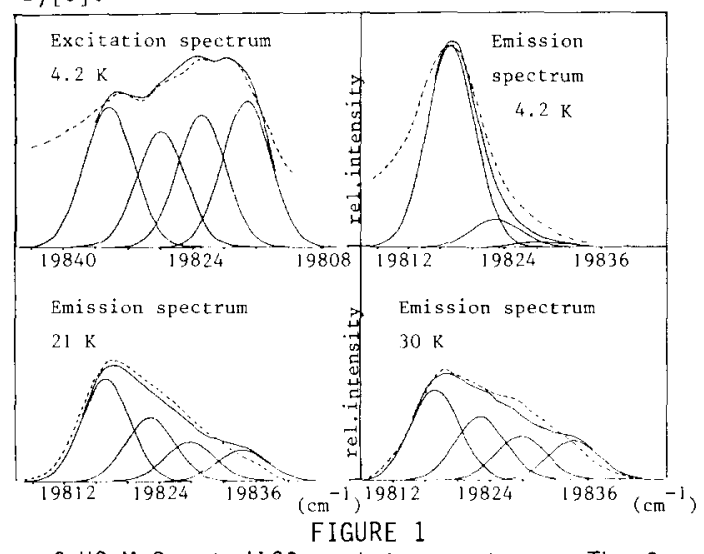

Zero-phonon $\mathrm{line}$ of $\mathrm{UO}_{2} \mathrm{MOO}_{4}$ at different temperatures. The Gaussians represent the correlation field components which constitute the observed line. Braken line: observed curve, solid line: sum curve. The positions of the Gaussians are: $19817.3,19822.9,19827.9$ and $19834.7 \mathrm{~cm}^{-1}$.

The intrinsic emission shows a non-single exponential decay, $\tau=0.1>3 \mu \mathrm{s}$. About $10 \mu \mathrm{s}$ after the pulse the traps attain to maximum intensity. The trap lines show a single-exponential decay, $\tau=60-90 \mu \mathrm{s}$. The temperature dependence of $T$ for traps 5 and 6 follows a decay function based upon a three leve 1 system (trap 5) [4] or a four-level (trap 6) system as shown in figs. 2 and 3. The final expressions are for trap 5:

$$
\tau_{\text {eff }}^{-1}=\left[w_{21}+w_{t i} \exp (-\Delta E / k T)\right][1+\exp (-\Delta E / k T)]^{-1}
$$

and for trap 6:

$$
{ }^{-1} \text { eff }=\left[w_{21}+w_{31} \exp (-\Delta \varepsilon / k T)+w_{t i} \exp (-\Delta E / k T)\right][1+\exp (-\Delta \varepsilon / k T)]^{-1}
$$

A detailed discussion of Eqs.(1) and (2) will be given in a forthcoming paper [5]. Using the experimental values $\tau_{21}(\mathrm{t} 5)=88 \mu \mathrm{s}, \Delta \mathrm{E}(\mathrm{t} 5)=185 \mathrm{~cm}^{-1}, \tau_{21}(\mathrm{t} 6)=$ $60 \mu \mathrm{s}, \Delta E(t 6)=272 \mathrm{~cm}^{-1}$ the $\tau(T)$ values could be fitted with $\tau_{t j}(t 5)=2.50$ $10^{-10} \mathrm{~s}, \tau_{31}(\mathrm{t} 6)=31.5 \mu \mathrm{s}, \Delta \varepsilon=39 \mathrm{~cm}^{-1}, \tau_{t i}(t 6)=2.1710^{-10} \mathrm{~s}$. Estimating the hopping time $\tau_{i j}$ between intrinsic centres from $\tau_{i i} \approx h / \Delta E_{C F}[6]$, where $\Delta E_{C F}$ denotes the correlation field splitting, we find with $\Delta \mathrm{E}_{\mathrm{CF}} \approx \approx 10 \mathrm{~cm}^{-1}$ (see caption fig. 1) [3] for $\tau_{i j} \approx 3.3310^{-12} \mathrm{~s}$. Other estimates $[7,8]$ are in the order of $10^{-8}-10^{-9} \mathrm{~s}$. Roughly, ${ }^{\mathrm{T}} i i$ and ${ }_{t} \mathrm{ti}$ seem to be of the same order of magnitude indicating an energy transfer proces intermediate between trap-1imited migration and diffusion-limited migration. 


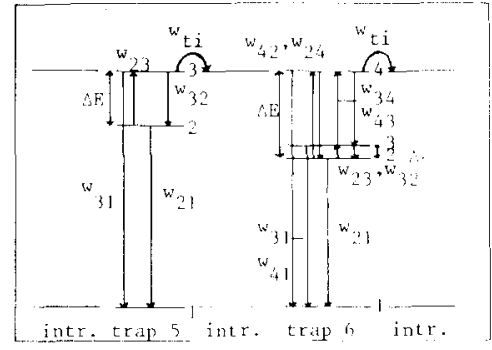

FIGURE 2

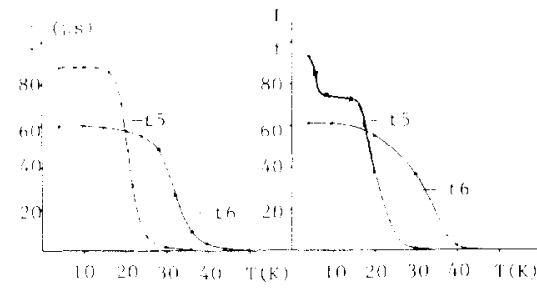

FIGURE 3

Schematic energy level diagram for in- $T$ vs. T and I vs. T curves for traps 5 trinsic centres and traps 5 and 6 . The and 6 . The full lines for the : vs. T transition probabilities Wij taken into curves are the final fitting curves obaccount in the derivation of the decay tained from Eqs. $(1,2)$. The full 1 ines functions for the trap levels are indi-for the I vs. T curves are drawn by cated in the figure. hand through the experimental points (indicated in both figures by crosses).

\section{REFERENCES}

1) V.N. Serezhkin, V.K. Trunov and L.G. Makarevich, Sov. Phys. Crystallogr., 25 (1980) 492.

2) R.G. Denning, T.R. Snellgrove and D.R. Woodwark, Mol. Phys., 37 (1979) 1109.

3) W.M.A. Smit and G. Blasse, J. Lumin., 29 (1984), in press.

4) B. di Bartolo, Optical Interactions in Solids, Wiley, New York, 1968, pg 445.

5) W.M.A. Smit and G. Blasse, to be published.

6) H.C. Wolf in Advances in Atomic and Molecular Physics, Vol. 3, pg 119, D.R. Bates and I. Estermann, Eds., Acad. Press, New York, 1967.

7) N.A. Tolstoi, A.P. Abramov and I.N. Abramova, J. Lumin., 1/2 (1970) 330.

8) D.M. Krol and A. Roos, Phys. Rev., B 23 (1981) 2135. 